\title{
Perspectivas de los criterios de evaluación financiera, una selfie al presupuesto de proyectos de inversión
}

\section{Perspectives of financial evaluation criteria, a selfie to the budget of investment projects}

\author{
Carlos Omar Soto González \\ José Kennedy Ollague Valarezo \\ Vicente Arias Montero \\ Carlos Sarmiento Chugcho \\ Universidad Técnica de Machala, Ecuador
}

Autor para correspondencia: csoto@utmachala.edu.ec, jollague@utmachala.edu.ec, varias@utmachala.edu.ec

Fecha de recepción: 02 de Agosto de 2017 - Fecha de aceptación: 10 de Agosto de 2017

Resumen: El objetivo de ésta investigación, es identificar las necesidades de información de los procesos de evaluación financiera de los presupuestos de proyectos de inversión, mediante la aplicación de la técnica de encuestas, con la finalidad de proponer una metodología adecuada para el proceso de enseñanza-aprendizaje de la aplicación de los criterios de evaluación financiera. Se aplica el método de aprendizaje basado en estudio de casos, el método deductivo e inductivo, el enfoque de la metodología desarrollada es cuantitativa - descriptiva y la modalidad de investigación es de tipo bibliográfica. Los resultados son obtenidos a través del programa estadístico "SPSS" en la cual se obtienen las frecuencias y los coeficientes de correlación de las variables de Pearson y Spearman, cuya significa se interpreta de manera satisfactoria debido que la hipótesis es positiva, por ello, se propone una metodología que favorezca el proceso enseñanza - aprendizaje, respecto al cálculo de criterios de evaluación financiera.

Palabras clave: presupuesto de capital; riesgo; rendimiento; recuperación de la inversión

\begin{abstract}
The objective of this research is to propose a methodology of the teaching-learning process of financial evaluation criteria related to net present value, internal rate of return and period of recovery of the investment; Through the combination of theoretical foundations and the praxis that gathers all the information with steps to be carried out to evaluate an investment project in a way that is friendly to the teacher and student. We apply the learning method based on case study, the deductive and inductive methods, the methodology approach developed is quantitative descriptive and the research modality is of a bibliographic type. The overall results are satisfactory, based on a methodology that favors the teaching - learning process, regarding the calculation of financial evaluation criteria.
\end{abstract}

Key words: capital budget; risk; return; investment recovery 


\section{Introducción}

En el tiempo actual, es común observar personas, analizando la posibilidad de invertir su dinero en la ejecución de un proyecto o idea de negocio, para esto el inversionista que no quiere arriesgar su capital debe efectuar una estimación que le muestre la conveniencia económica del proyecto antes de realizar la inyección de sus recursos, esta estimación se realiza en base a estudios previos de la idea, que le permitirán pronosticar y analizar cada una de las variables tales como los posibles ingresos y egresos que envuelven todo el proyecto.

El estudio previo a la inversión consiste en un resumen de la información pronosticada que ayudará a determinar los flujos netos de cada período de ejecución del proyecto, cabe mencionar que los períodos se expresan generalmente en años y la cantidad de años a utilizarse dependerá de la naturaleza de cada proyecto o idea.

Todo ese contexto empresarial y financiero, para que sea de éxito, necesita de un aporte significativo que se genera en las aulas universitarias, y en relación a éste tema, existe la problemática que para el aprendizaje de los estudiantes respecto a evaluar financieramente proyectos de inversión, los documentos bibliográficos de muy buena calidad, referente a éste tema lo tratan de manera general, en la explicación del ¿cómo se soluciona un determinado ejercicio o caso? Especialmente lo relacionado al criterio de evaluación que tiene que ver con la tasa interna de rendimiento, debido que para docentes y estudiantes en ciertas circunstancias se le presenta complejo el proceso, por ello optan por utilizar en una hoja de cálculo la opción de funciones; pero es importante en un educando de nivel superior conozca holísticamente el proceso evaluación financiera con los cálculos del valor actual neto, tasa interna de rendimiento y período de recuperación de la inversión.

El objetivo de la investigación es identificar las necesidades de información de los procesos de evaluación financiera de los presupuestos de proyectos de inversión, mediante la aplicación de la técnica de encuestas, con la finalidad de proponer una metodología adecuada para el proceso de enseñanza-aprendizaje de la aplicación de los criterios de evaluación financiera relacionados al valor actual neto, tasa interna de rendimiento y período de recuperación de la inversión.

En toda investigación es imperativo e importante mencionar las opiniones o criterios de investigadores entendidos en el ámbito empresarial y financiero, por ello bien menciona, Andía Valencia (2011), para realizar un proyecto de inversión es fundamental identificar el problema, el paso siguiente es cuantificar la necesidad de recursos, motivo por el cual es importante determinar correctamente la demanda insatisfecha del mismo. Los proyectos de inversión deben corresponder a las necesidades de un conglomerado, con la finalidad de obtener el rendimiento estimado.

En todo tipo de inversión los factores de rendimientos van acompañados automáticamente de las posibilidades técnicas del riesgo, por ello, los autores Francischetti, Bertassi, Souza, Padoveze, \& Calil (2014) mencionan que, la estructura del mercado genera riesgos en todo momento, por lo tanto es importante encontrar el equilibrio entre la protección del capital y la inversión con el fin de alcanzar un mayor beneficio económico. 
La toma de decisiones inmersa en la administración financiera, fija su objetivo principal en el valor de la decisiones de inversión, tomando en consideración tres puntos importantes: valor del dinero en el tiempo, fuente de financiamiento y costo medio ponderado del capital (Altuve, 2004).

Toda empresa u organización, se enfoca en mejorar la calidad de su servicio o producto, es en esta parte en donde necesita dirigir sus proyectos de inversión, por lo cual necesita herramientas financieras para estimar sus posibles resultados (Andia Valencia, 2010). Las herramientas, instrumentos o criterios de evaluación financiera, contribuyen en la toma de decisiones adecuadas, con la finalidad de determinar los posibles rendimientos y minimizar el riesgo en las inversiones.

También los proyectos se valoran considerando su tasa de recuperación y su rendimiento, las cuales se estiman a raíz de sus entradas de efectivo (Canales Salinas, 2015). Estas entradas y salidas de efectivo se establecen en el instrumento cuantitativo y sistemático de los estudios del proyecto, denominado presupuesto de capital o presupuesto de proyectos de inversión.

El presupuesto de capital incluye las inversiones, ingresos, costos y gastos del proyecto; pero también los proyectos de inversión presentan gastos de depreciación y a la inflación como impuestos implícitos, los cuales no logran igualar el valor presente del costo del uso del capital. (Ávila Mahecha \& León Hernández, 2010).

Los proyectos de inversión son evaluados a raíz de la proyección de flujo de fondos y su tasa de descuento, es importante destacar que las ventas y gastos definen la ecuación financiera de las empresas (Dapena \& Alonso, 2015). Uno de los parámetros de mayor importancia en la evaluación de proyectos es, la tasa de descuento, ya que a través del tiempo compara los flujos de beneficios (Edwards, 2016). Esta tasa de descuento permite traer a valor presente los flujo futuros sean positivos o negativos, que se han obtenido a través de la estructuración del presupuesto de capital.

Existen diferentes maneras de evaluar un proyecto, encontrándose entre las principales, el valor actual neto (VAN) y la tasa interna de rendimiento (TIR), necesitando de los flujos de caja y tasa de corte como datos fundamentales para el análisis del mismo (Mavila Hinojoza \& Polar Falcon, 2005). La evaluación financiera de los proyectos es analizada a través del descuento de los flujos de efectivo, el VAN y la TIR, a partir de esto se puede tomar o rechazar alguna propuesta (Mete, 2014).

En similar sentido (Valencia, 2014), menciona que, los proyectos de inversión se evalúan indicadores de rentabilidad como el VAN (Valor actual neto), la TIR (Tasa interna de retorno); además del EVA (Valor económico agregado), estos indicadores son muy importantes para la implementación de estrategias y posterior logro de objetivos planteados. Según Milanesi (2016), un método alternativo para analizar el rendimiento promedio de las inversiones es la Tasa Interna de Retorno promedio borrosa (TIRP) a través de esta se puede resolver los problemas que la TIR presenta frente al valor actual (VA). 
Otras opiniones sustentan que, los proyectos de inversión son evaluados generalmente por tres parámetro: Proyección de flujo de fondos, tasa de descuento y VAN (valor actual neto) (Zúñiga-Jara, Soria, \& Sjoberg, 2011). Entre los criterios de evaluación financiera que no se considera en ésta investigación por estar enfocada al sector social, es el análisis coste-beneficio que es la principal herramienta para la evaluación económica en la administración de recursos, además es importante tener una tasa social de descuento para valorar los proyectos de interés social (Cruz \& Muñoz, 2007).

El desarrollo de los países se fomentan mediante la política estatal, y una de las recomendaciones es generar el incremento de las inversiones mediante la iniciativa de los emprendimiento organizacionales, tal como lo mencionan, Cardona Montoya, Martins, \& Velásquez Ceballos (2017), que el emprendimiento corporativo genera ventajas competitivas y dinamiza el crecimiento de las economías a través de su entorno macroeconómico. Aunque en los últimos años las economías en desarrollo han recibido grandes capitales, debido al lento crecimiento de los países desarrollados, enfocándose en la seguridad alimentaria y ambiental (Salas Couce, 2014). Tanto los países desarrollados y en vías de desarrollo, tienen la necesidad de fomentar la inversión a todo nivel, debido a las situaciones para equilibrar sus presupuestos estatales.

Los inconvenientes que se presentan en las iniciativas de emprendimientos y sus posibles inversiones, es el financiamiento y como financiar sus proyectos; por ello tradicionalmente optan por financiarse o apalancarse de la forma tradicional, pero al generarse un apalancamiento de tipo financiero, tiene sus efectos, tal como lo mencionan, Castillo-Merino, Menéndez-Plans, \& Orgaz-Guerrero (2013), que el apalancamiento financiero tiene un efecto positivo en el costo de capital de la empresas al aumentar la tasa de descuento de los flujos de efectivo, además que la adopción de las NIFF ha llevado a cabo un menor coste de capital de las mismas.

Por la alta competencia que se genera a partir de la investigación e innovación, la inversión en capital humano es fundamental en una economía, ya que a mayor inversión en este campo mayor productividad, por ende, mayor incentivo salarial o viceversa; además marca una diferencia entre los diferentes actores de este mercado. (Gómez \& Ramírez, 2015). Adicionalmente los sistemas financieros sufren transformaciones debido al desarrollo tecnológico y de innovación financiera, por tal motivo los proyectos de inversión son verdaderos desafíos para el mercado bursátil (Rodríguez, 2007).

Los autores Miralles-Marcelo, Miralles-Quirós, \& Miralles-Quirós (2011) mencionan que, en los últimos años los mercados financieros han decaído, por tal motivo la investigación en este campo ha incrementado considerablemente para determinar sus efectos; además demuestran que la inversión en pequeñas y medianas empresas obtienen mayor rentabilidad.

Los proyectos de desarrollo son fundamentales para la economía de una nación, por un lado el sector público que fomenta la redistribución de riqueza de un país y por la otra vía, el sector privado, que genera la productividad del mismo, lo óptimo es mantener un equilibrio adecuado (Ruiz Tibana \& Duarte, 2015). Si se mantiene una armonía en las inversiones, le genera a un estado mayor liquidez y dinamismo a su economía. 
Sin embargo, existen algunas consideraciones en el contexto de las inversiones, y esto lo sustenta, Duque Oliva \& Báez Roa (2012), que las reformas tributarias pueden contribuir a las empresas a mejorar sus flujos de caja o disminuir su costo de capital, a través del impuesto de transacciones financieras. Es importante los, incentivos tributarios, que permiten incrementar los flujos de recursos a través del aumento de las inversiones. A nivel internacional, en ciertas circunstancias es necesario el arbitraje de inversión que resuelve de manera alternativa los problemas que los tratados bilaterales cometen en el flujo de bienes y servicio en el entorno internacional (Segura España, 2014).

Según, Santillán, Martínez, \& López (2016) mencionan que una mayor intervención financiera permite desarrollar la actividad real de la economía de un país, al aumentar la liquidez y disminuir el costo de oportunidad de la inversión. La inyección de recursos por el sistema financiero a una tasa de interés baja, genera la expectativa de solicitudes de crédito y por ende aumenta la producción y consumo de bienes y servicios.

Las alternativas conceptuales mencionadas en ésta fundamentación, respecto a las inversiones que nacen de los emprendimientos, que para ejecutar una inversión se requiere de un apalancamiento y para establecer si un proyecto es viable o factible, se necesita de un instrumento denominado presupuesto de proyectos de inversión que es largo plazo, tal como menciona, (Useche Arévalo, 2014), que las decisiones de presupuesto de capital generan mayor impacto para el largo plazo de las empresas, que están basadas en las proyecciones y comportamientos futuros, variables que son de incertidumbre dentro de la economía de un país.

A través del análisis económico se mejora la toma de decisiones en los proyectos de inversión para optimizar los índices de productividad (Márquez Díaz, Castro, \& Julián, 2015). El análisis económico, se sustenta en los criterios de evaluación financiera que se son el valor actual neto, tasa interna de retorno y período de recuperación de la inversión, que se aplica a la información del presupuesto de capital o presupuesto de proyectos de inversión.

El direccionamiento que tomará el presente documento, está enfocado a dar solución a la problemática y a cumplir con el objetivo propuesto; por ello se presenta ésta metodología de proceso de enseñanza aprendizaje que contiene la secuencia de la solución del presupuesto de capital que es insumo imperativo para el proceso de cálculo del valor actual neto, tasa interna de retorno y período de recuperación de la inversión. El método aplicado es estudio de casos en conjunto con el deductivo e inductivo y finalmente se presentan las conclusiones, a partir de la hipótesis de la investigación: "Las insuficientes metodologías para la elaboración y cálculo del presupuesto de capital, valor actual neto, tasa interna de rendimiento y período de recuperación de la inversión, influye en el proceso de enseñanza - aprendizaje en los estudiantes de educación superior".

\section{Métodos}

La investigación tiene el enfoque cualitativo, descriptivo y cuantitativo. Se sustenta en el método de aprendizaje basado en estudio de casos en conjunto con los métodos deductivo e inductivo. 
Es de tipo cualitativa, debido que la presente investigación se aplica el instrumento de encuestas con el objetivo de identificar las necesidades de información respecto al proceso de evaluación financiera de los presupuestos de proyectos de inversión, en los estudiantes de séptimo nivel de la carrera de contabilidad y auditoría, en la Universidad Técnica de Machala; es decir mediante ésta encuesta se identifica la problemática y necesidad de contar con una guía o metodología amigable para poder calcular los criterios de evaluación financiera, como el valor actual neto, tasa interna de rendimiento y período de recuperación de la inversión en relación al presupuesto de capital o presupuesto de proyectos de inversión, que muchas ocasiones se le dificulta al estudiante determinar la viabilidad financiera de un proyecto, mediante la aplicación del VAN, TIR y PRI.

Se aplica la encuesta al cien por ciento de los estudiantes matriculados en los séptimos niveles de la sección matutina y nocturna en el período lectivo Mayo a Septiembre 2017, que son total 130, de las cuales 126 estudiantes contestaron el cuestionario. Esta información recopilada se procesa a través del programa estadístico informático "SPSS", cuyos resultados de las frecuencias y la medición del grado de significancia a través de los coeficientes de correlación de las variables de Spearman y de Pearson; y se reflejan en tablas y gráficos.

Es de tipo descriptiva, porque se interpreta los resultados de la investigación que se reflejan en las tablas y gráficos procesados por el programa estadístico "SPSS", adicionalmente la información de tipo cuantitativa que se obtiene a través del método de aprendizaje basado en estudio de casos; también se analiza los resultados del proceso de elaboración del presupuesto de proyecto de inversión, valor actual neto, tasa interna de retorno y período de recuperación de la inversión, que son datos numéricos, son sujetos de interpretación para determinar la viabilidad financiera de un proyecto.

La modalidad de la investigación es de tipo bibliográfica - documental, en el marco de los criterios de evaluación financiera de proyectos de inversión y de campo debido al levantamiento de información mediante las encuestas.

\section{Resultados}

La información de la investigación procesada por el programa estadístico informático "SPSS", proporciona los siguientes resultados por cada interrogante del cuestionario.

1. ¿La temática del presupuesto de capital o presupuesto de proyecto de inversión con que asignatura se relaciona?

En base al criterio del estudiante, el presupuesto de proyecto de inversión se relaciona con la Administración Financiera y Presupuesto (23,02\%), con Administración Financiera, Presupuesto y Elaboración de Proyectos de Inversión (23,02\%), el 23,02\% de los encuestados, respondió que el presupuesto de proyecto de inversión se relaciona con todas las asignaturas, es decir con Administración Financiera, Presupuesto, Emprendimiento y Elaboración de Proyectos de Inversión. 
2. ¿Los criterios de evaluación financiera, como el valor actual neto (VAN), tasa interna de rendimiento (TIR), período de recuperación de la inversión (TIR), en que asignatura los ha estudiado o está estudiando?

En esta pregunta del cuestionario, el 39,68\% de los estudiantes respondieron que la temática de los criterios de evaluación financiera, como el valor actual neto, tasa interna de rendimiento y período de recuperación de la inversión, la han estudiado en las asignaturas de Administración Financiera, Presupuesto y Emprendimiento. El 25,40\% respondió que estudió estos temas en Administración Financiera y Presupuesto, mientras que el 7,94\% respondió que las materias de Administración Financiera y Emprendimiento. Es decir las asignaturas de Emprendimiento, Presupuesto y Administración Financiera ya las han analizado en el proceso de estudio hasta séptimo nivel, por ello se aplica la investigación en éste nivel de estudio.

3. ¿En qué nivel considera usted, que le resultó resolver un caso o ejercicio de presupuesto de capital o presupuesto para proyectos de inversión?

El 46,03\% de los encuestados respondieron que se le resultó complicado de resolver el presupuesto de capital, mientras que el $44,44 \%$ opina que se le hizo fácil de resolver, mientras que el 3,175\% opina que se le hizo muy fácil, 3,175\% muy complicado.

4. ¿En qué nivel considera usted, que le resultó calcular el criterio de evaluación financiera, valor actual neto - VAN?

En relación a la investigación, el 63,49\% de los encuestados opina que le resultó fácil, calcular el valor actual, mientras que el $20,63 \%$ opina que le resultó complicado, el 15,08\% muy fácil, y el 0,794\% muy complicado.

5. ¿En qué nivel considera usted, que le resultó calcular el criterio de evaluación financiera, tasa interna de rendimiento - TIR?

Para el cálculo de la tasa interna de rendimiento, según los encuestados, al 43,65\% le resultó fácil, al 37,30\% le pareció complicado, el 9,524\% no conoce el proceso para calcular, mientras que el 7,143\% le resultó muy fácil de resolver.

6. ¿En qué nivel considera usted, que le resultó calcular el criterio de evaluación financiera, período de recuperación de la inversión? 


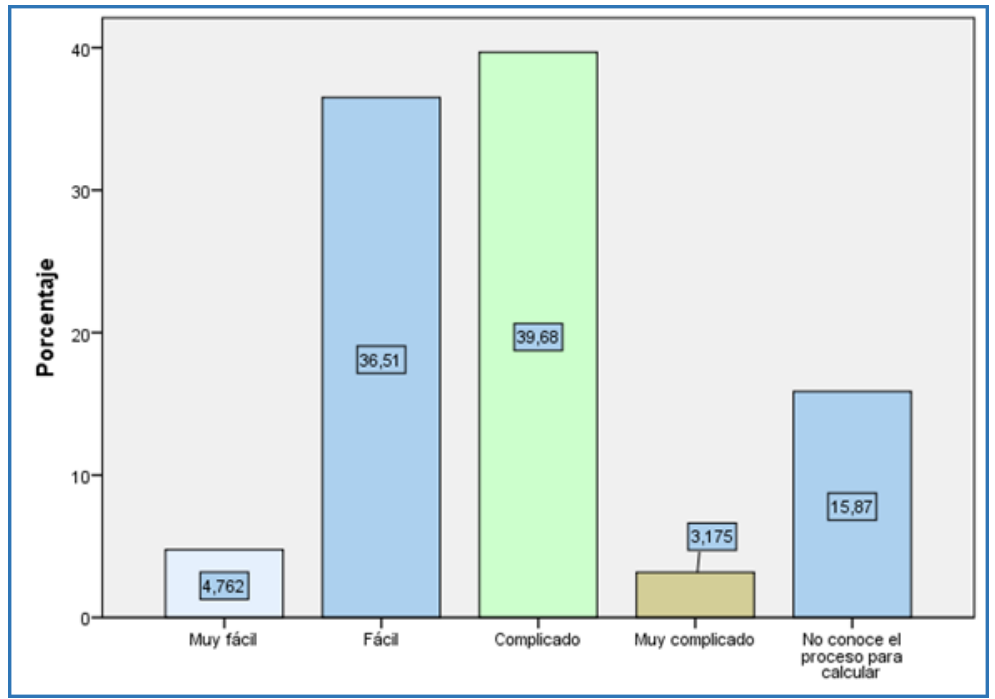

Figura 1: Nivel de complejidad de resolución del PRI.

\section{Análisis del resultado de la pregunta 6.}

Para el cálculo del período de recuperación de la inversión, el 39,68\% opina que le resultó complicado, al 36,51\% menciona que le resultó fácil, el 15,87\% no conoce el proceso para calcular, mientras que al 4,76\% y al 3,18\% menciona que se le hizo muy fácil y muy complicado respectivamente.

1. ¿En caso de haber existido, cuáles fueron los motivos para que tenga dificultad en elaborar el presupuesto de capital y calcular el VAN, TIR y el período de recuperación de la inversión?

Respecto a ésta pregunta, para identificar las dificultades que se presentan al elaborar el presupuesto de capital y calcular el valor actual neto, tasa interna de rendimiento, período de recuperación de la inversión, el 46,03\% de los encuestados opinan que no cuenta con un procedimiento fácil de entender, como guía para calcular; el 8,73\% menciona que no cuenta con fuentes de consulta amigables; para el 7,94\% opina que la bibliografía no cumple con las expectativas de los encuestados en relación a la temática. Mientras que el 0,79\% menciona que hasta el momento no conoce del tema y el 36,51\% restante opina que son otros los motivos por lo que se dificulta elaborar y hacer los cálculos de la temática en proceso de investigación.

1. ¿En qué nivel fueron amigables o accesibles, las fuentes bibliográficas que ha investigado, para calcular los criterios de evaluación financiera (VAN - TIR - PRI)?

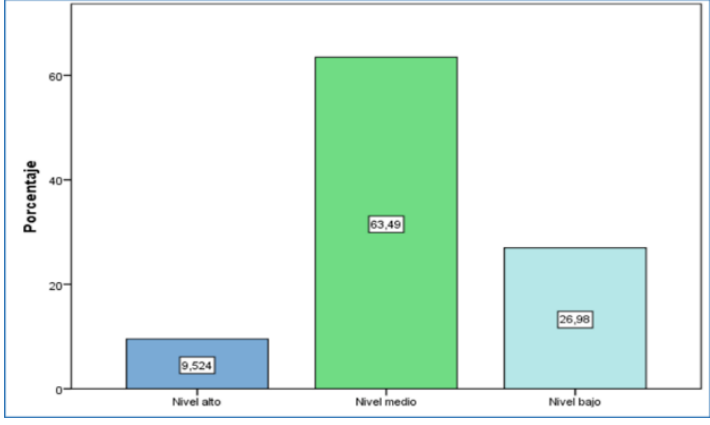

Figura 2: Nivel de información de fuentes bibliográficas 


\section{Análisis del resultado de la pregunta 8.}

En base a los resultados, los encuestados opinan que las fuentes bibliográficas que han investigado para calcular el valor actual neto, la tasa interna de rendimiento y el período de recuperación de la inversión, les pareció amigables o útiles para resolver, el 63,49\% opina que en un nivel medio; el 26,98\% en un nivel bajo; y el 9,52\% piensa que en un nivel alto.

2. ¿Ha calculado el VAN y el TIR, en una hoja de cálculo con la opción funciones financieras?

El 54,76\% de los encuestados mencionan que no han calculado el valor actual neto y la tasa interna de rendimiento en una hoja electrónica, mientras que el 45,24\% afirma que ha realizado las operaciones financieras con la opción funciones financieras.

3. ¿Ha calculado la tasa interna de rendimiento en forma manual?

Del total de encuestados el 76,98\% menciona que si ha calculado la tasa interna de rendimiento en forma manual, mientras que el $23,02 \%$ menciona que no ha calculado el criterio de evaluación financiera.

4. ¿Cuál es el motivo que no ha calculado la tasa interna de rendimiento en forma manual?

Respecto a los motivos que mencionan los encuestados para no haber calculado la tasa interna de rendimiento en forma manual, el 54,76\% no contestó esta pregunta, debido a que en alguna ocasión, ya lo había calculado, el 16,67\% menciona que le resultó complicado calcular, el $13,49 \%$ no conoce el proceso, el 11,11\% no entiende el proceso, mientras que el 3,97\% no conoce una guía o proceso para calcular.

2. ¿Si hubiere un método o procedimiento amigable y accesible para elaborar y calcular el presupuesto de capital, el valor actual neto, la tasa interna de rendimiento y el período de recuperación de la inversión, publicado en un artículo de revista científica, usted lo utilizaría como fuente de investigación y estudio? 


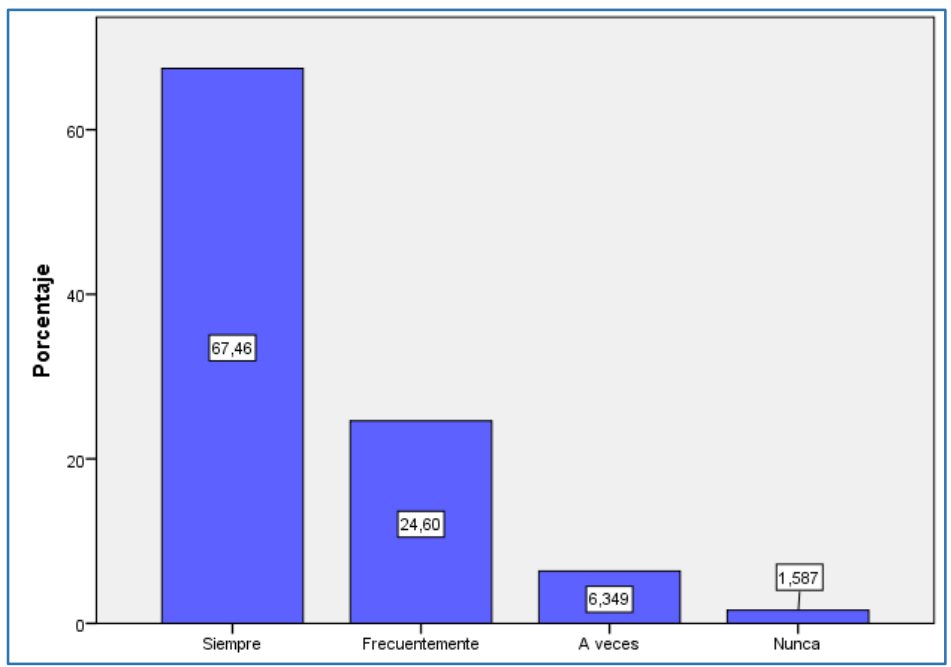

Figura 3: Nivel de posible utilización de artículo académico

\section{Análisis del resultado de la pregunta 12.}

En esta interrogante, el 67,46\% de los encuestados mencionan que utilizarían siempre si hubiere un método y procedimiento amigable y accesible para elaborar y calcular el presupuesto de capital, el valor actual neto, la tasa interna de rendimiento, y período de recuperación de la inversión; el 24,60\% menciona que frecuentemente utilizaría, mientras que el 6,35\% a veces. Aquí se interpreta que existe la necesidad de contar con una guía o procedimiento que le permita al lector, calcular los criterios de evaluación financiera, de manera especial la tasa interna de retorno y el período de recuperación de la inversión.

Tabla 1. Correlación de variables de Pearson

\begin{tabular}{|c|c|c|c|c|c|c|c|}
\hline \multicolumn{2}{|c|}{ Variables } & \multirow{3}{*}{$\begin{array}{c}\text { Nivel de } \\
\text { complejidad } \\
\text { de la TIR }\end{array}$} & \multirow{2}{*}{$\begin{array}{c}\text { Nivel de } \\
\text { complejidad } \\
\text { del PRI } \\
, 332^{* *}\end{array}$} & \multirow{2}{*}{$\begin{array}{c}\text { Motivos } \\
\text { para no } \\
\text { cálculo } \\
\text { del PC y } \\
\text { CEV } \\
-, 062\end{array}$} & \multirow{2}{*}{$\begin{array}{c}\text { Nivel de las } \\
\text { fuentes } \\
\text { bibliográfica. }\end{array}$} & \multirow{2}{*}{$\begin{array}{l}\text { Cálculo } \\
\text { del } \\
\text { VAN y } \\
\text { TIR } \\
, 291^{* *}\end{array}$} & \multirow{2}{*}{$\begin{array}{c}\begin{array}{c}\text { Nivel de } \\
\text { utilización } \\
\text { de } \\
\text { metodología }\end{array} \\
, 062\end{array}$} \\
\hline Nivel de & Correlación & & & & & & \\
\hline $\begin{array}{c}\text { complejidad } \\
\text { en solucionar } \\
\text { De la TIR }\end{array}$ & $\begin{array}{c}\text { de Pearson } \\
\text { Sig. } \\
\text { (bilateral) }\end{array}$ & & , 000 & 491 & ,000 & , 001 & ,488 \\
\hline & $\mathrm{N}$ & 126 & 126 & 126 & 126 & 126 & 126 \\
\hline $\begin{array}{c}\text { Nivel de } \\
\text { complejidad }\end{array}$ & $\begin{array}{c}\text { Correlación } \\
\text { de Pearson }\end{array}$ &, $332^{* *}$ & 1 & ,033 &, $230^{* *}$ & , 140 &,- 043 \\
\hline $\begin{array}{c}\text { en solucionar } \\
\text { El PRI }\end{array}$ & $\begin{array}{c}\text { Sig. } \\
\text { (bilateral) }\end{array}$ & 000 & & ,715 &, 010 & ,118 & ,629 \\
\hline & $\mathrm{N}$ & 126 & 126 & 126 & 126 & 126 & 126 \\
\hline $\begin{array}{l}\text { Motivos para } \\
\text { no calcular el }\end{array}$ & $\begin{array}{c}\text { Correlación } \\
\text { de Pearson }\end{array}$ &,- 062 & ,033 & 1 &,- 067 &,- 029 &,- 002 \\
\hline $\begin{array}{l}\text { Presupuesto } \\
\text { de Capital y }\end{array}$ & $\begin{array}{c}\text { Sig. } \\
\text { (bilateral) }\end{array}$ & ,491 & ,715 & & ,459 & ,745 & ,982 \\
\hline $\begin{array}{c}\operatorname{los} \\
\text { Criterios de }\end{array}$ & $\mathrm{N}$ & 126 & 126 & 126 & 126 & 126 & 126 \\
\hline $\begin{array}{c}\text { Evaluación F. } \\
\text { Nivel de ser } \\
\text { amigables las }\end{array}$ & $\begin{array}{c}\text { Correlación } \\
\text { de Pearson }\end{array}$ &, $331^{* *}$ &, $230^{* *}$ &,- 067 & 1 & ,192* &,- 146 \\
\hline
\end{tabular}




\begin{tabular}{|c|c|c|c|c|c|c|c|}
\hline $\begin{array}{c}\text { Fuentes } \\
\text { bibliográficas }\end{array}$ & $\begin{array}{c}\text { Sig. } \\
\text { (bilateral) }\end{array}$ & ,000 & ,010 & ,459 & & ,032 & , 103 \\
\hline & $\mathrm{N}$ & 126 & 126 & 126 & 126 & 126 & 126 \\
\hline $\begin{array}{l}\text { Cálculo del } \\
\text { valor actual }\end{array}$ & $\begin{array}{l}\text { Correlación } \\
\text { de Pearson }\end{array}$ & ,291** &, 140 &,- 029 &, $192^{*}$ & 1 & ,116 \\
\hline $\begin{array}{c}\text { neto } \\
\mathrm{Y} \text { tasa interna }\end{array}$ & $\begin{array}{c}\text { Sig. } \\
\text { (bilateral) }\end{array}$ & ,001 &, 118 &, 745 & ,032 & & , 195 \\
\hline de retorno & $\mathrm{N}$ & 126 & 126 & 126 & 126 & 126 & 126 \\
\hline $\begin{array}{l}\text { Nivel de } \\
\text { posible }\end{array}$ & $\begin{array}{l}\text { Correlación } \\
\text { de Pearson }\end{array}$ & ,062 &,- 043 &,- 002 &,- 146 &, 116 & 1 \\
\hline $\begin{array}{l}\text { utilización } \\
\text { De la }\end{array}$ & $\begin{array}{c}\text { Sig. } \\
\text { (bilateral) }\end{array}$ & 488, & ,629 & 982 &, 103 & ,195 & \\
\hline $\begin{array}{c}\text { metodología } \\
\text { propuesta }\end{array}$ & $\mathrm{N}$ & 126 & 126 & 126 & 126 & 126 & 126 \\
\hline
\end{tabular}

\section{En la correlación de Pearson, se interpreta.}

- Nivel de complejidad en solucionar la tasa interna de rendimiento con nivel de complejidad en solucionar el período de recuperación de la inversión, tiene un valor de $\mathrm{p}$ de $1 \%$.

- Nivel de complejidad en solucionar la tasa interna de rendimiento con el nivel de ser amigables o accesibles para el cálculo de las fuentes bibliográficas tiene un valor de $\mathrm{p}$ de $1 \%$.

- Nivel de complejidad en solucionar la tasa interna de rendimiento con cálculo del VAN y TIR en una hoja electrónica, tiene un valor de $\mathrm{p}$ de $1 \%$.

- Nivel de complejidad en solucionar el período de recuperación de la inversión con nivel de ser amigables o accesibles para el cálculo de las fuentes bibliográficas, tiene un valor de $\mathrm{p}$ de $1 \%$.

- Motivos para no calcular el presupuesto de capital y los criterios de evaluación financiera con Motivos del no cálculo de la TIR tiene un valor de p de 5\%.

- Nivel de fuentes bibliográficas con cálculo del VAN y TIR tiene un valor de p de 5\%.

Tabla 2. Correlación de variables de Spearman

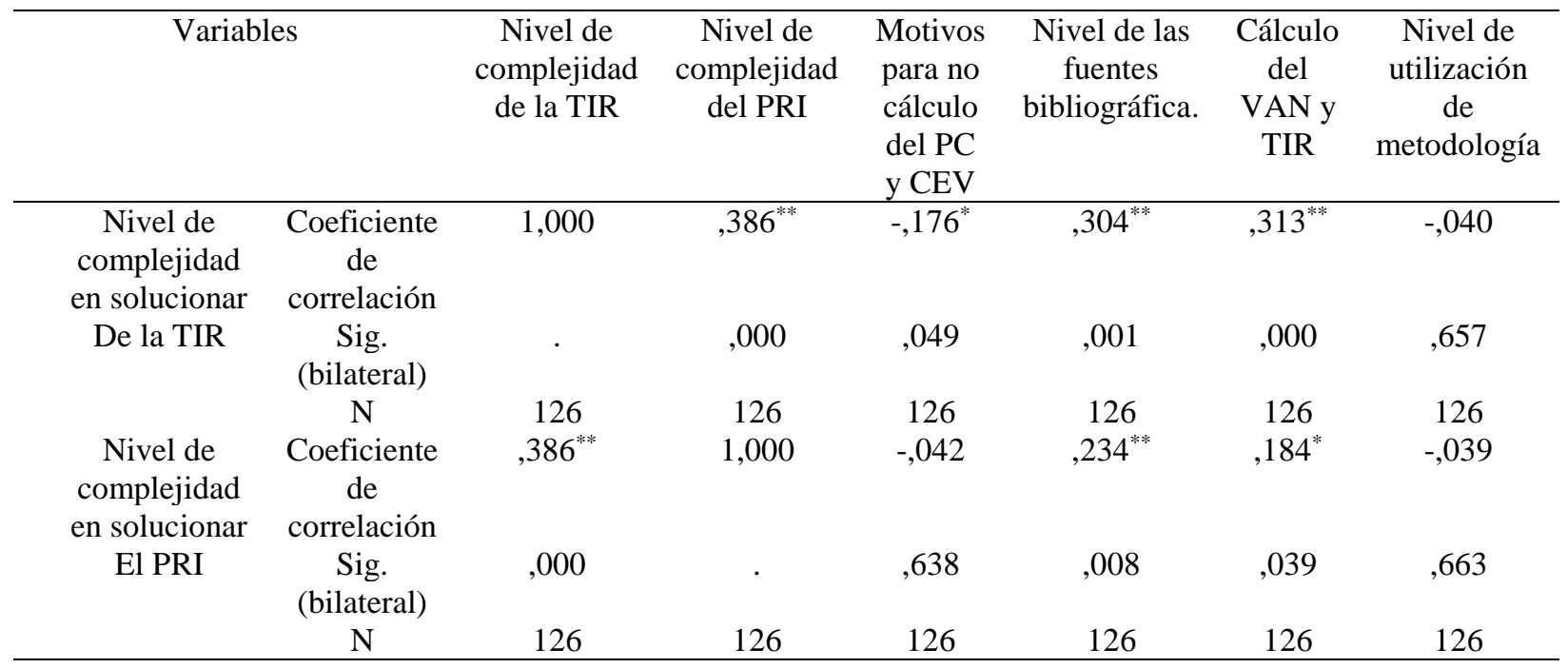




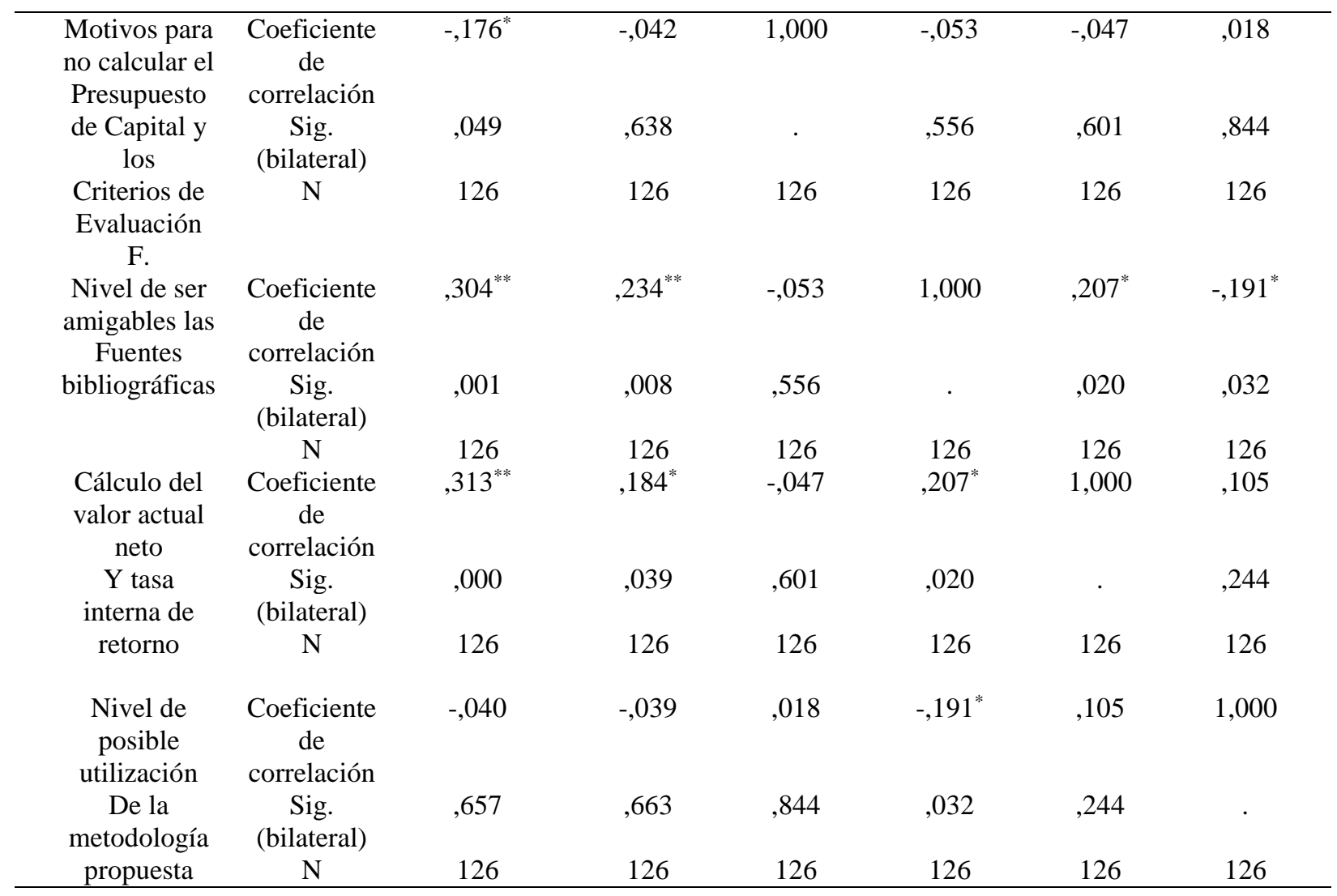

\section{En la correlación de Spearman:}

- Nivel de solución del TIR con Nivel de solución del PRI tiene un valor de p de $1 \%$.

- Nivel de solución del TIR. con Motivos para calcular PC y criterios EVA tiene un valor de $\mathrm{p}$ de $5 \%$.

- Nivel de solución del TIR con Calculo del VAN y TIR tiene un valor de p de $1 \%$

- Nivel de solución del TIR. con Nivel de fuentes bibliográficas tiene un valor de p de 5\%.

- Nivel de solución del PRI con Nivel de fuentes bibliográficas tiene un valor de p de $1 \%$.

- Nivel de solución del PRI. con Calculo del VAN y TIR tiene un valor de p de 5\%.

- Nivel de fuentes bibliográficas con Calculo del VAN y TIR tiene un valor de p de 5\%.

- Nivel de fuentes bibliográficas con Método proceso amigable tiene un valor de p de

\section{Discusión}

En base a los resultados obtenidos de la investigación se interpreta mediante las preguntas de la encuesta y los niveles de las frecuencias de cada una de ellas que en efecto existen inconvenientes y les resulta complejo a los estudiantes, calcular la tasa interna de rendimiento, período de recuperación promedio y se evidencia que es necesario para los estudiantes contar con una metodología que se accesible y amigable para entender y calcular los criterios de evaluación financiera tales como el valor actual neto, tasa interna de rendimiento y período de recuperación de la inversión. 
Adicionalmente se demuestra estadísticamente que la hipótesis es positiva, mediante la correlación de las variables Pearson y Spearman cuya interpretación se describen en los resultados del presente documento. En tal sentido, se demuestra que existe una alta correlación ya que el p asociado es del $1 \%$ y $5 \%$ respectivamente, en donde se puede determinar que las variables escogidas para la explicación de este estudio tienen un nivel de significancia altamente aceptable.

En relación a los resultados e interpretaciones antes especificados, y en concordancia al objetivo de la investigación se propone el siguiente procedimiento para el cálculo del valor actual neto, tasa interna de rendimiento, período de recuperación de la inversión, que son los criterios de evaluación financiera que se aplica al presupuesto de proyectos de inversión.

El proceso para calcular los criterios de evaluación financiera en un proyecto de inversión es el siguiente.

1. Fijar el proyecto de inversión a realizarse para efectos de estudio o enseñanza.

2. Elaborar el presupuesto de capital, con los datos del proyecto de inversión en los siguientes componentes: Flujo de Inversión, Flujo de Operación y Flujo Neto.

Tabla 3. Flujo de Inversión del Presupuesto de Capital

\begin{tabular}{|c|c|c|c|c|c|c|c|c|c|}
\hline \multirow{10}{*}{ 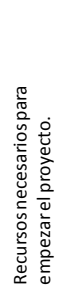 } & & \multirow[b]{2}{*}{ Inversión inicial } & \multicolumn{6}{|c|}{ FLUJO DE INVERSIÓN } & \\
\hline & & & \multicolumn{6}{|c|}{ Período del proyecto } & \multirow{9}{*}{ 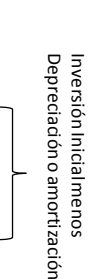 } \\
\hline & CONCEPTO & 0 & 1 & 2 & 3 & 4 & & 5 & \\
\hline & TERRENOS & $\$ \quad(1,000,000.00)$ & & & & & $\$$ & $1,000,000.00$ & \\
\hline & CONSTRUCCIONES & $\$ \quad(2,000,000.00)$ & & & & & $\$$ & $1,525,000.00$ & \\
\hline & MAQUINARIA Y EQUIPO 1 & $\$ \quad(2,000,000.00)$ & & & & & $\$$ & $1,100,000.00$ & \\
\hline & \begin{tabular}{|l} 
MOBILIARIO 1 \\
\end{tabular} & $\begin{array}{ll}\$ & (800,000.00) \\
\end{array}$ & & & & & $\$$ & $288,000.00$ & \\
\hline & COSTO Y ESTUD. PROYECTO & 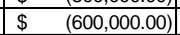 & & & & & $\$$ & & \\
\hline & \begin{tabular}{|l|l|} 
CAPITAL DE TRABAJO \\
\end{tabular} & $\$(1,400,000.00)$ & & & & & $\$$ & $1,400,000.00$ & \\
\hline & TOTAL DE INVERSIÓN & $\$(7,800,000.00)$ & & & & & $\$$ & $5,313,000.00$ & \\
\hline
\end{tabular}

Tabla 4: Depreciaciones de activos del Proyecto

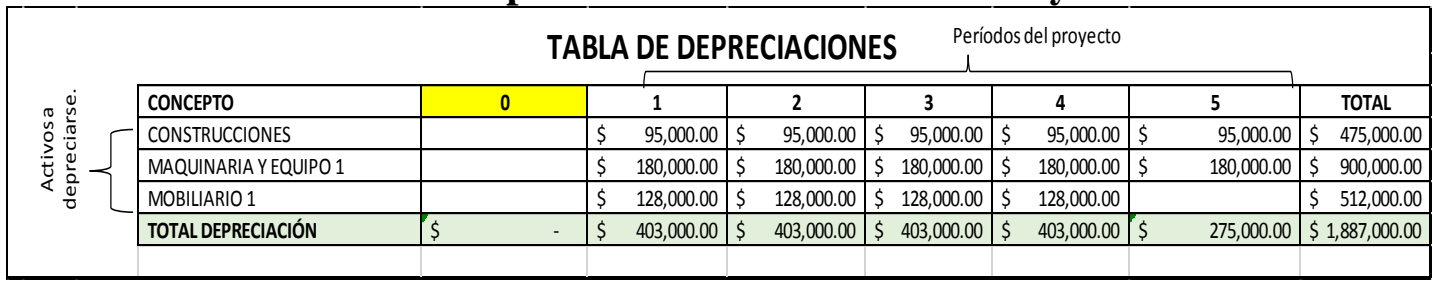

Tabla 5: Amortizaciones del Proyecto

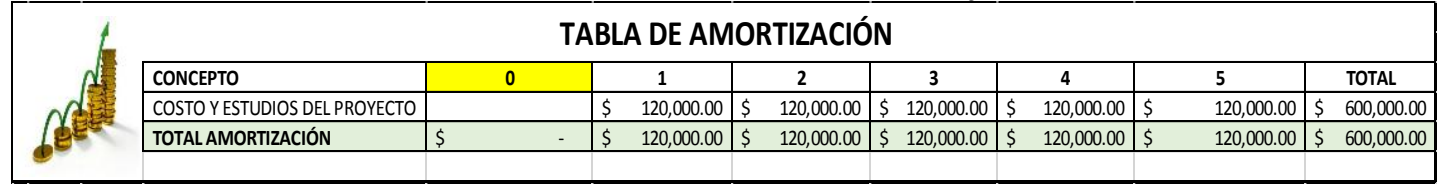

Tabla 6: Flujo Operativo del Presupuesto de Capital 


\begin{tabular}{|c|c|c|c|c|c|c|c|}
\hline \multicolumn{8}{|c|}{ FLUJO DE OPERACIÓN } \\
\hline CONCEPTO & 0 & 1 & 2 & 3 & 4 & & 5 \\
\hline \multicolumn{8}{|l|}{ INGRESOS } \\
\hline VENTAS & & $\$ 9,800,000.00$ & $\$ 9,800,000.00$ & $\$ 9,800,000.00$ & $\$ 14,000,000.00$ & $\$$ & $14,000,000.00$ \\
\hline TOTAL INGRESOS & & $\$ 9,800,000.00$ & $\$ 9,800,000.00$ & $\$ 9,800,000.00$ & $\$ 14,000,000.00$ & $\$$ & $14,000,000.00$ \\
\hline \multicolumn{8}{|l|}{ EGRESOS } \\
\hline \multicolumn{8}{|l|}{ COSTOS } \\
\hline MATERIA PRIMA Y MAT. DIRECTOS & & $\$ 1,400,000.00$ & $\$ 1,400,000.00$ & $\$ 1,400,000.00$ & $\$ 2,000,000.00$ & $\$$ & $2,000,000.00$ \\
\hline MANO DE OBRA & & $\$ 4,200,000.00$ & $\$ 4,200,000.00$ & $\$ 4,200,000.00$ & $\$ 6,000,000.00$ & $\$$ & $6,000,000.00$ \\
\hline TOTAL COSTOS & & $\$ 5,600,000.00$ & $\$ 5,600,000.00$ & $\$ 5,600,000.00$ & $\$ 8,000,000.00$ & $\$$ & $8,000,000.00$ \\
\hline \multicolumn{8}{|l|}{ GASTOS } \\
\hline SALARIOS & & $\$ 2,000,000.00$ & $\$ 2,000,000.00$ & $\$ 2,000,000.00$ & $\$ 2,000,000.00$ & $\$$ & $2,000,000.00$ \\
\hline SERVICIOS & & $280,000.00$ & $\$ \quad 280,000.00$ & $\$ 280,000.00$ & $\$ \quad 280,000.00$ & $\$$ & $280,000.00$ \\
\hline MANTENIMIENTO & & $100,000.00$ & $\$ 100,000.00$ & $\$ 100,000.00$ & $\$ 100,000.00$ & $\$$ & $100,000.00$ \\
\hline SEGUROS & & $90,000.00$ & $90,000.00$ & $\$ \quad 90,000.00$ & $90,000.00$ & $\$$ & $90,000.00$ \\
\hline OTROS GASTOS & & $130,000.00$ & $130,000.00$ & $\$ 130,000.00$ & $130,000.00$ & $\$$ & $130,000.00$ \\
\hline DEPRECIACIÓN & & $\$ \quad 403,000.00$ & $403,000.00$ & $\$ 403,000.00$ & $403,000.00$ & $\$$ & $275,000.00$ \\
\hline AMORTIZACIÓN & & $120,000.00$ & $\$ \quad 120,000.00$ & $\$ 120,000.00$ & $120,000.00$ & $\$$ & $120,000.00$ \\
\hline TOTAL GASTOS & & $\$ 3,123,000.00$ & $\$ 3,123,000.00$ & $\$ 3,123,000.00$ & $\$ 3,123,000.00$ & $\$$ & $2,995,000.00$ \\
\hline TOTAL EGRESOS & & $\$ 8,723,000.00$ & $\$ 8,723,000.00$ & $\$ 8,723,000.00$ & $\$ 11,123,000.00$ & $\$$ & $10,995,000.00$ \\
\hline UTILIDAD & & $\$ 1,077,000.00$ & $\$ 1,077,000.00$ & $\$ 1,077,000.00$ & $\$ 2,877,000.00$ & $\$$ & $3,005,000.00$ \\
\hline PART. TRABAJADORES & & $\$ \quad 161,550.00$ & $\$ \quad 161,550.00$ & $\$ 161,550.00$ & $\$ \quad 431,550.00$ & $\$$ & $450,750.00$ \\
\hline IMPUESTOS & & $201,399.00$ & $201,399.00$ & $\$ 201,399.00$ & $\$ \quad 537,999.00$ & $\$$ & $561,935.00$ \\
\hline UTILIDAD NETA & & $714,051.00$ & $714,051.00$ & $\$ 714,051.00$ & $\$ 1,907,451.00$ & $\$$ & $1,992,315.00$ \\
\hline DEPRECIACIÓN & & $\$ \quad 403,000.00$ & $403,000.00$ & $\$ 403,000.00$ & $\$ \quad 403,000.00$ & $\$$ & $275,000.00$ \\
\hline AMORTIZACIÓN & & $120,000.00$ & $120,000.00$ & $\$ 120,000.00$ & $120,000.00$ & $\$$ & $120,000.00$ \\
\hline EXCEDENTE O DÉFICIT & & $\$ 1,237,051.00$ & $\$ 1,237,051.00$ & $\$ 1,237,051.00$ & $\$ 2,430,451.00$ & $\$$ & $2,387,315.00$ \\
\hline
\end{tabular}

Tabla 7: Resultados finales del Presupuesto de Capital

\begin{tabular}{|c|c|c|c|c|c|c|c|c|c|}
\hline \multicolumn{10}{|c|}{ FLUJO NETO } \\
\hline CONCEPTO & & 0 & & 1 & 2 & 3 & 4 & & 5 \\
\hline TOTAL INVERSIÓN & $\$$ & $(7,800,000.00)$ & $\$$ & - & $\$$ & $\$$ & $\$$ & $\$$ & $5,313,000.00$ \\
\hline EXCEDENTE O DEFICIT & $\$$ & - & $\$$ & $1,237,051.00$ & $\$ 1,237,051.00$ & $\$ 1,237,051.00$ & $\$ 2,430,451.00$ & $\$$ & $2,387,315.00$ \\
\hline FLUJO NETO FINANCIERO & $\$$ & $(7,800,000.00)$ & $\$$ & $1,237,051.00$ & $\$ 1,237,051.00$ & $\$ 1,237,051.00$ & $\$ 2,430,451.00$ & $\$$ & $7,700,315.00$ \\
\hline
\end{tabular}

1. Aplicar los criterios de evaluación financiera para medir la viabilidad del proyecto.

\section{Valor Actual Neto}

\section{Fórmula:}

$I \emptyset=$ Inversión inicial

$$
\text { Valor actual neto }(V A N)=I \emptyset \pm \frac{F_{1}}{(1+i)^{n}}+\frac{F_{2}}{(1+i)^{n}} \ldots
$$

$\boldsymbol{F}_{\mathbf{1}=\text { flujo neto periodo } 1}$

$\mathrm{i}=$ Tasa De Descuento

$\mathrm{n}=$ Periodo

Ejemplo:

Con la información de los flujos netos del presupuesto de capital en constan en el numeral 2, se determina si el proyecto es viable desde el punto de vista del VAN, para éste proceso se aplica como referencia una tasa de descuento del $10 \%$.

Paso 1: Identificar los flujos netos en el presupuesto de capital.

\section{Tabla 8. Flujo Neto Financiero}




\begin{tabular}{|l|l|l|l|l|l|l|l|l|l|}
\hline FLUJONETOFINANCIERO & $\$(7,800,000,00$ & $\$ 1,237,051,00$ & $\$ 1,237,051,00$ & $\$ 1,237,051,00$ & $\$ 2,430,451,00$ & $\$$ & $\$ 1,700,315,00$ \\
\hline
\end{tabular}

Paso 2: Reemplazar la fórmula con los datos correspondientes y realizar los cálculos. Valor actual neto $(V A N)=I \emptyset \pm \frac{F_{1}}{(1+i)^{n}}+\frac{F_{2}}{(1+i)^{n}} \ldots$

$$
\begin{aligned}
\boldsymbol{V} \boldsymbol{A} \boldsymbol{N}=-7^{\prime} 800.000,00 \pm \frac{1^{\prime} 237.051,00}{(1+0.10)^{1}}+\frac{1^{\prime} 237.051,00}{(1+0.10)^{2}}+\frac{1^{\prime} 237.051,00}{(1+0.10)^{3}}+\frac{2^{\prime} 430.451,00}{(1+0.10)^{4}} \\
+\frac{7^{\prime} 700.315,00}{(1+0.10)^{5}}
\end{aligned}
$$

Paso 3: Colocar el mismo valor en la inversión inicial y resolver el denominador de cada flujo.

$$
\begin{aligned}
\boldsymbol{V} \boldsymbol{A} \boldsymbol{N}=-7^{\prime} 800.000,00 \pm \frac{1^{\prime} 237.051,00}{1.10}+\frac{1^{\prime} 237.051,00}{1.21}+\frac{1^{\prime} 237.051,00}{1.331}+\frac{2^{\prime} 430.451,00}{1.4621} \\
+\frac{7^{\prime} 700.315,00}{1.6105}
\end{aligned}
$$

Paso 4: Colocar el mismo valor en la inversión inicial y dividir cada uno de los numeradores con los denominadores.

$$
\begin{gathered}
\boldsymbol{V A N =}-7^{\prime} .800 .000 \pm 1^{\prime} 124.591,82+1^{\prime} 022.356,20+929.414,73+1^{\prime} 662.301,48 \\
+4^{\prime} 781.319,47
\end{gathered}
$$

Paso 5: Restar la inversión inicial con la sumatoria total de los flujos.

$$
\begin{gathered}
\boldsymbol{V A N}=-7^{\prime} 800.000+9^{\prime} 519.983,69 \\
\boldsymbol{V A N}=\$ 1^{\prime} 719.983,69 \boldsymbol{V I A B L E}
\end{gathered}
$$

\section{Tasa Interna De Retorno}

Fórmula:

Tasa Interna De Retorno $(T I R)=I n+(I m-I n)\left[\frac{(V A N+)}{(V A N+)-(V A N-)}\right]$

In $=$ Tasa de descuento menor

$\mathbf{I m}=$ Tasa de descuento mayor

VAN $+=$ Valor actual neto positivo

VAN - = Valor actual neto negativo

Ejemplo:

Con la información de los flujos netos del presupuesto de capital del numeral 2, se determina si el proyecto es viable desde el punto de vista de la TIR. 
Paso 1: Verificar el VAN obtenido, con los datos anteriores; si el VAN es positivo se debe buscar el VAN negativo, y si el VAN calculado es negativo se debe buscar el positivo.

Para este ejemplo, el VAN que se ha calculado previamente, es positivo; por ello se busca el VAN negativo.

- Para encontrar el VAN negativo se debe elevar la tasa de descuento, en relación a la tasa de descuenta aplicada para calcular el VAN positivo. Al mismo tiempo que la tasa de descuento de la va incrementando gradualmente, el resultado del VAN, tendrá que ir disminuyendo hasta convertirse en negativo.

- La tasa de descuento a utilizarse es la primera con la cual el VAN se convierte en negativo, lo más aproximado a cero.

$$
\begin{aligned}
& \text { Valor actual neto }(V A N)=I \emptyset \pm \frac{F_{1}}{(1+i)^{n}}+\frac{F_{2}}{(1+i)^{n}} \ldots \\
& \boldsymbol{V A N}=-7^{\prime} 800.000,00 \pm \frac{1^{\prime} 237.051,00}{(1+0.16)^{1}}+\frac{1^{\prime} 237.051,00}{(1+0.16)^{2}}+\frac{1^{\prime} 237.051,00}{(1+0.16)^{3}}+\frac{2^{\prime} 430.451,00}{(1+0.16)^{4}} \\
& +\frac{7^{\prime} 700.315,00}{(1+0.16)^{5}} \\
& \boldsymbol{V} \boldsymbol{A N}=-7^{\prime} 800.000,00 \pm \frac{1^{\prime} 237.051,00}{1.16}+\frac{1^{\prime} 237.051,00}{1.3456}+\frac{1^{\prime} 237.051,00}{1.5609}+\frac{2^{\prime} 430.451,00}{1.8106} \\
& +\frac{7^{\prime} 700.315,00}{2.1003} \\
& \boldsymbol{V} \boldsymbol{A} \boldsymbol{N}=-7^{\prime} 800.000,00+1^{\prime} 066.423,28+919.330,41+792.524,18+1^{\prime} 342.345,63 \\
& +3{ }^{\prime} 666.292,91 \\
& \boldsymbol{V} \boldsymbol{A N}=-7^{\prime} 800.000,00+7^{\prime} 786.916,41 \\
& V A N=\$-13.083,59
\end{aligned}
$$

Paso 2: Reemplazar La Fórmula Con Los Datos Correspondientes.

\section{Datos:}

In = Tasa de descuento menor $\mathbf{1 0 \%}$

$\mathbf{I m}=$ Tasa de descuento mayor $\mathbf{1 6 \%}$

VAN + =Valor actual neto positivo \$1'719.983,69

VAN - = Valor actual neto negativo $\$ \mathbf{- 1 3 . 0 8 3 , 5 9}$

Tasa Interna De Retorno $($ TIR $)=t n+(t m-t n)\left[\frac{(V A N+)}{(V A N+)-(V A N-)}\right]$

Tasa Interna De Retorno (TIR)

$$
=0.10+(0.16-0.10)\left[\frac{\left(1^{\prime} 719.983,69\right)}{\left(1^{\prime} 719.983,69\right)-(13.083,59)}\right]
$$

Paso 3: Resolver paréntesis y corchetes.

Tasa Interna De Retorno $(T I R)=0.10+(0.06)(1.0077)$ 


\section{Tasa Interna De Retorno $(T I R)=0.1605 * 100$ \\ $T I R=16.05 \%$ VIABLE}

\section{Período De Recuperación De La Inversión}

Paso 1: Establecer una línea de tiempo de los flujos netos del presupuesto.

Tabla 9. Línea de tiempo de flujos netos

\begin{tabular}{c|cccccc}
\hline Determinación & $\mathbf{0}$ & $\mathbf{1}$ & $\mathbf{2}$ & $\mathbf{3}$ & $\mathbf{4}$ & $\mathbf{5}$ \\
\hline & $(7.800 .000,00$ & $1.237 .051,0$ & $1.237 .051,0$ & $1.237 .051,0$ & $2.430 .451,0$ & $7.700 .315,0$ \\
Flujo Neto & ) & 0 & 0 & 0 & 0 & 0 \\
\hline
\end{tabular}

Paso 2: Determinar los flujos netos acumulados.

Se inicia desde el período 1, el flujo neto acumulado es el mismo valor del flujo neto del período 1. Para obtener el resultado del flujo neto acumulado del periodo 2 , se suma el flujo neto acumulado del periodo 1 con el flujo neto del periodo 2; y así sucesivamente para el resto de periodos del proyecto.

Tabla 10. Flujo neto acumulado

\begin{tabular}{l|cccccc}
\hline Determinación & $\mathbf{0}$ & $\mathbf{1}$ & $\mathbf{2}$ & $\mathbf{3}$ & $\mathbf{4}$ & $\mathbf{5}$ \\
\hline Flujo Neto & $(7.800 .000,00)$ & $1.237 .051,00$ & $1.237 .051,00$ & $1.237 .051,00$ & $2.430 .451,00$ & $7.700 .315,00$ \\
& & & & & & \\
$\begin{array}{l}\text { Flujo Neto } \\
\text { Acumulado }\end{array}$ & & $1.237 .051,00$ & $2.474 .102,00$ & $3.711 .153,00$ & $6.141 .604,00$ & $13.841 .919,00$ \\
\hline
\end{tabular}

Paso 3: Identificar y ubicar la inversión inicial en los rangos que corresponda dentro de la línea de tiempo de los flujos netos acumulados.

Tabla 11. Rango de la Inversión Inicial

\begin{tabular}{|c|c|c|c|c|c|c|}
\hline Determinación & 0 & 1 & 2 & 3 & 4 & 5 \\
\hline Flujo Neto & )$^{(7.800 .000,00}$ & $\begin{array}{l}1.237 .051,0 \\
0\end{array}$ & $\begin{array}{l}1.237 .051,0 \\
0\end{array}$ & $\begin{array}{l}1.237 .051,0 \\
0\end{array}$ & $\begin{array}{l}2.430 .451,0 \\
0\end{array}$ & $7.700 .315,00$ \\
\hline $\begin{array}{l}\text { Flujo Neto } \\
\text { Acumulado }\end{array}$ & & $\begin{array}{l}1.237 .051,0 \\
0\end{array}$ & $\begin{array}{l}2.474 .102,0 \\
0\end{array}$ & $\begin{array}{l}3.711 .153,0 \\
0\end{array}$ & $0 \underbrace{6.14} \underbrace{1.604,0}$ & $\int_{0}^{13.841 .919,0}$ \\
\hline & & & & & \multicolumn{2}{|c|}{$\begin{array}{l}\text { Rango de inversión } \\
\text { inicial. }\end{array}$} \\
\hline
\end{tabular}

Paso 4: Una vez ubicada la inversión inicial en el rango correspondiente, al periodo o año base se la denominara flujo neto acumulado inferior y ese se considerara el tiempo de recuperación en años. El período o año que cierra el rango se lo denomina flujo neto acumulado superior.

Tabla 12: Período de recuperación en años

\begin{tabular}{l|llllll}
\hline Determinación & $\mathbf{0}$ & $\mathbf{1}$ & $\mathbf{2}$ & $\mathbf{3}$ & $\mathbf{4}$ & $\mathbf{5}$ \\
\hline
\end{tabular}




\begin{tabular}{|c|c|c|c|c|c|c|}
\hline Flujo Neto & $\begin{array}{l}(7.800 .000,00 \\
)\end{array}$ & $\begin{array}{l}1.237 .051,0 \\
0\end{array}$ & $\begin{array}{l}1.237 .051,0 \\
0\end{array}$ & $\begin{array}{l}1.237 .051,0 \\
0\end{array}$ & $\begin{array}{l}2.430 .451,0 \\
0\end{array}$ & $7.700 .315,00$ \\
\hline $\begin{array}{l}\text { Flujo Neto } \\
\text { Acumulado }\end{array}$ & & $\begin{array}{l}1.237 .051,0 \\
0\end{array}$ & $\begin{array}{l}2.474 .102,0 \\
0\end{array}$ & $\begin{array}{l}3.711 .153,0 \\
0\end{array}$ & $\begin{array}{l}6.141 .604,0 \\
0\end{array}$ & $\begin{array}{l}13.841 .919,0 \\
0\end{array}$ \\
\hline & & \multicolumn{2}{|c|}{$\begin{array}{l}\text { F. ACUM. } \\
\text { INFERIOR }\end{array}$} & $\begin{array}{c}\$ \\
7^{\prime} 800.000 .00\end{array}$ & \multicolumn{2}{|c|}{$\begin{array}{r}\text { F. ACUM. } \\
\text { SUPERIOR }\end{array}$} \\
\hline
\end{tabular}

Paso 5: Para encontrar el periodo de recuperación en meses, se tiene que buscar un valor que vaya al numerador y otro al denominador y para ello, se resta la inversión inicial, con el flujo neto acumulado inferior, luego se resta el flujo neto acumulado superior con el inferior.

$$
\begin{aligned}
& \text { Período De Recuperación Meses }=\frac{(\text { Inv. Inic. }- \text { F. Acum. Inferior })}{(\text { F. Acum. Superior }- \text { F. Acum. Inferior })} \\
& \text { Período De Recuperación Meses }=\frac{\left(7^{\prime} 800.000,00 .-6^{\prime} 141.604,00\right)}{\left(13^{\prime} 841.919,00-6^{\prime} 141.604,00\right)} \\
& \text { Período De Recuperación Meses }=\frac{\left(1^{\prime} 658.396,00\right)}{\left(7^{\prime} 700.315,00\right)}=\mathbf{0 , 2 1 5 3 6 7}
\end{aligned}
$$

Paso 6: El resultado que proporciona el paso 5, se multiplica por 12 para conocer los meses y los decimales del nuevo resultado por 30 para conocer los días.

$$
\text { Periodo Recuperación Meses = 0,215367 X12 * Pri Meses = 2,58 M. }
$$

Pri En Días = 0,58 X30 Pri En Días = 17,4 D.

Paso 7: Establecer el período de recuperación con la información obtenida de los cálculos.

Período de recuperación de la inversión = 4 años, 2 meses y 17 días.

\section{Conclusión}

La presente investigación permite concluir que el conocimiento para elaborar el presupuesto de capital, y aplicar los criterios de evaluación financiera, son fundamentales para determinar si un proyecto de inversión es viable o factible financieramente; y también es importante que el personal dicente cuente con una guía que le sirva como procedimiento idóneo para su aprendizaje, y no solo el estudiantes sino el profesional en el ámbito empresarial que necesite de herramientas financieras para su adecuada aplicación. Por ello se ha propuesto en ésta investigación un proceso factible y amigable para la correcta aplicación de los criterios de evaluación financiera.

\section{Bibliografía}


Altuve, J. G. (2004). El Uso Del Valor Actual Neto Y La Tasa Interna De Retorno Para La Valoración De Las Decisiones De Inversión. Actualidad Contable Faces, 7(9), 7-17.

Andia Valencia, W. (2010). Proyectos De Inversión: Un Enfoque Diferente De Análisis. Revista De La Facultad De Ingeniería Industrial, 13(1), 028-031.

Andía Valencia, W. (2011). La Demanda Insatisfecha En Los Proyectos De Inversión Pública. Industrial Data, 14(2), 67-72.

Ávila Mahecha, J., \& León Hernández, I. (2010). Un Impuesto Implícito A La Inversión. Cuadernos De Economía, 29(52), 127-146.

Canales Salinas, R. J. (2015). Criterios Para La Toma De Decisión De Inversiones. REICE: Revista Electrónica De Investigación En Ciencias Económicas, 3(5), 101-117.

Cardona Montoya, R. A., Martins, I., \& Velásquez Ceballos, H. (2017). Entrepreneurial Orientation, Assessment and Management of Projects and Impact in Corporate Entrepreneurship: Intention to Action. Cuadernos De Gestión, 17(2), 37-60. Http://Doi.Org/10.5295/Cdg.140511rc

Castillo-Merino, D., Menéndez-Plans, C., \& Orgaz-Guerrero, N. (2013). Mandatory IFRS Adoption and the Cost of Equity Capital: Evidence from Spanish Firms. Intangible Capital, 10(3), 562-583. Http://Doi.Org/10.4090/Juee.2008.V2n2.033040

Cruz, S., \& Muñoz, M. (2007). Obtención De La Tasa Social De Descuento A Partir De La Tasa De Fallo De Una Distribución Estadística: Aplicación Empírica. Estudios De Economía Aplicada, 25(1), 49-82.

Dapena, J. P., \& Alonso, J. C. (2015). Aspectos Financieros En La Gestión De La Empresa Y En La Evaluación De Proyectos De Inversión. (No. 568). Econstor.

Duque Oliva, E. J., \& Báez Roa, M. D. P. (2012). Effect of Taxes on Capital Cost: Case Study of Almacenes Éxito S.A. (2006 -2010). Revista Facultad De Ciencias Económicas: Investigación Y Reflexión, 20(2), 205-229.

Edwards, G. (2016). Estimación De La Tasa Social De Descuento A Largo Plazo En El Marco De Los Sistemas Nacionales De Inversión. Trimestre Económico, 83(329), 99-125.

Francischetti, C. E., Bertassi, A. L., Souza, L., Padoveze, C., \& Calil, J. F. (2014). El Análisis De Riesgos Como Herramienta Para La Toma De Decisiones Relativas A Inversiones. Invenio, 17(33), 73-85.

Gómez, A. M., \& Ramírez, Z. (2015). Disparidades Salariales Y La Tasa Interna De Retorno A La Educación Privada En Los Docentes De La Universidad Del Cauca. Civilizar, 15(28), $165-180$. 
Márquez Díaz, C., Castro, M., \& Julián, F. (2015). Uso Del Valor Actual Neto, Tasa Interna De Retorno Y Relación Beneficio-Costo En La Evaluación Financiera De Un Programa De Vacunación De Fiebre Aftosa En El Estado Yaracuy, Venezuela. Revista De La Facultad De Ciencias Veterinarias, 56(1), 58-61.

Mavila Hinojoza, D., \& Polar Falcón, E. (2005). Flujo De Caja Y Tasa De Corte Para La Evaluación De Proyectos De Inversión. Industrial Data, 8(2).

Mete, M. R. (2014). Valor Actual Neto Y Tasa Interna De Retorno: Su Utilidad Como Herramientas Para El Análisis Y Evaluación De Proyectos De Inversión. Fides Et Ratio, 7(Marzo 2014), 67-85.

Milanesi, G. S. (2016). La Tasa Interna De Retorno Promedio Borrosa: Desarrollos Y Aplicaciones. Journal of Economics, Finance and Administrative Science, 21(40), 39-47. Http://Doi.Org/10.1016/J.Jefas.2015.12.001

Miralles-Marcelo, J. L., Miralles-Quirós, J. L., \& Miralles-Quirós, M. Del M. (2011). Rentabilidades Anormales Y Estrategias De Inversión En Periodos De Crisis: El Caso Español. Innovar, 21(39), 101-111.

Rodríguez, A. (2007). El Mercado De Capitales Como Alternativa De Inversión. Visión Gerencial, 6(Marzo 2007), 105-116.

Ruiz Tibana, M., \& Duarte, T. (2015). Los Proyectos De Desarrollo : La Inversión Pública Y La Inversión Privada. Scientia Et Technica, 20(2), 134-138.

Salas Couce, C. (2014). La Inversión Extranjera Directa En La Crisis. Economía Y Desarrollo, 151(1), 85-93.

Santillán, R., Martínez, M., \& López, F. (2016). Análisis Econométrico Del Riesgo Y Rendimiento De Las Siefores. Revista Mexicana De Economía Y Finanzas, 11(1), $29-54$.

Segura España, M. B. (2014). Arbitraje De Inversión : ¿Un Incentivo Para La Inversión Extranjera Directa? Revista De Derecho Privado, 51.

Useche Arévalo, A. J. (2014). Exceso De Confianza Y Optimismo En Las Decisiones De Presupuesto De Capital : Las Finanzas Corporativas Desde Un Enfoque Centrado En El Comportamiento. Universidad \& Empresa, 16(26), 93-114.

Valencia, W. A. (2014). Indicador De Rentabilidad De Proyectos: El Valor Actual Neto (VAN) O El Valor Económico Agregado (EVA). Industrial Data, 14(1), 015. Http://Doi.Org/10.15381/Idata.V14i1.6204

Zúñiga-Jara, S., Soria, K., \& Sjoberg, O. (2011). Costo Del Capital Y Evaluación De Proyectos En Latinoamérica: Una Clarificación. Innovar, 21(41), 39-49. 